\title{
The evaluation of nanoparticle-ligand distributions to
}

\section{determine nanoparticle concentration}

\author{
Uvaraj Uddayasankar, Ravi T. Shergill and Ulrich J. Krull* \\ Department of Chemical and Physical Sciences, University of Toronto Mississauga, 3359 \\ Mississauga Road, Mississauga, ON, Canada L5L1C6 \\ *E-mail: ulrich.krull@utoronto.ca, Fax: 9055694388
}

\begin{abstract}
The concentration of nanoparticles in solution is an important, yet challenging parameter to quantify. In this work, a facile strategy for the determination of nanoparticle concentration is presented. The method relies on the quantitative analysis of the inherent distribution of nanoparticle-ligand conjugates that are generated when nanoparticles are functionalized with ligands. Validation of the method was accomplished by applying it to gold nanoparticles and semiconductor nanoparticles (CdSe/ZnS; core/shell). Poly(ethylene glycol) based ligands, with functional groups that quantitatively react with the nanoparticles, were incubated with the nanoparticles at varying equivalences. Agarose gel electrophoresis was subsequently used to separate and quantify the nanoparticleligand conjugates of varying valences. The distribution in the nanoparticle-ligand conjugates agreed well with that predicted by the Poisson model. A protocol was then developed, where a series of only eight different ligand amounts could provide an estimate of the nanoparticle concentration that spans three orders of magnitude $(1 \mu \mathrm{M}$ to $1 \mathrm{mM})$. For the gold nanoparticles and semiconductor nanoparticles, the measured concentrations were found to deviate by only $7 \%$ and $2 \%$, respectively, from those
\end{abstract}


determined by UV-Vis spectroscopy. The precision of the assay was evaluated, resulting in a coefficient of variation of $5-7 \%$. Finally, the protocol was used to determine the extinction coefficient of alloyed semiconductor nanoparticles $\left(\mathrm{CdS}_{x} \mathrm{Se}_{1-x} / \mathrm{ZnS}\right)$, for which a reliable estimate is currently unavailable, at three different emission wavelengths (525, 575 and $630 \mathrm{~nm}$ ). The extinction coefficient of the nanoparticles at all emission wavelengths was similar, and was found to be $2.1 \times 10^{5} \mathrm{M}^{-1} \mathrm{~cm}^{-1}$.

\section{Introduction}

Nanomaterials have had a profound impact in many areas of analytical chemistry, and continue to be a driving force for the development of novel sensing schemes ${ }^{1}$. The utility of nanoparticles is a result of their unique properties that arise as a consequence of reducing the material size to nanometer dimensions. The implementation of nanoparticles in many different fields has necessitated the development of numerous techniques for their characterization and these have been recently reviewed ${ }^{2,3}$. Even with the multitude of methods available for the characterization of nanoparticles, the concentration of nanoparticles is often cited as a parameter that is challenging to determine ${ }^{4,5}$.

Nanoparticles do not have a defined molecular weight and this is a consequence of the variability of composition, dispersity in size and the distributions of arrangements of stabilizing ligands that cover the nanoparticles. The most common method of concentration determination relies on the combined use of ICP-AES and TEM imaging to compute the nanoparticle concentration ${ }^{6}$. The limitation of this method arises from the dispersity of nanoparticle sizes and shapes, which prevents the accurate determination of nanoparticle dimensions. The emerging use of nanomaterials with complex shapes and mixed compositions further increases the complexity in the determination of nanoparticle 
concentration using this method. Recognizing these challenges, some methods have been proposed for the determination of nanoparticle concentration including the use of single nanoparticle ICP-MS techniques ${ }^{7}$, electrospray droplet differential mobility analysis ${ }^{8}$ and single molecule particle tracking ${ }^{9}$. In this manuscript, we propose a novel approach that is readily available in most laboratories based on the determination of the inherent distribution of ligands on the surface of nanoparticles.

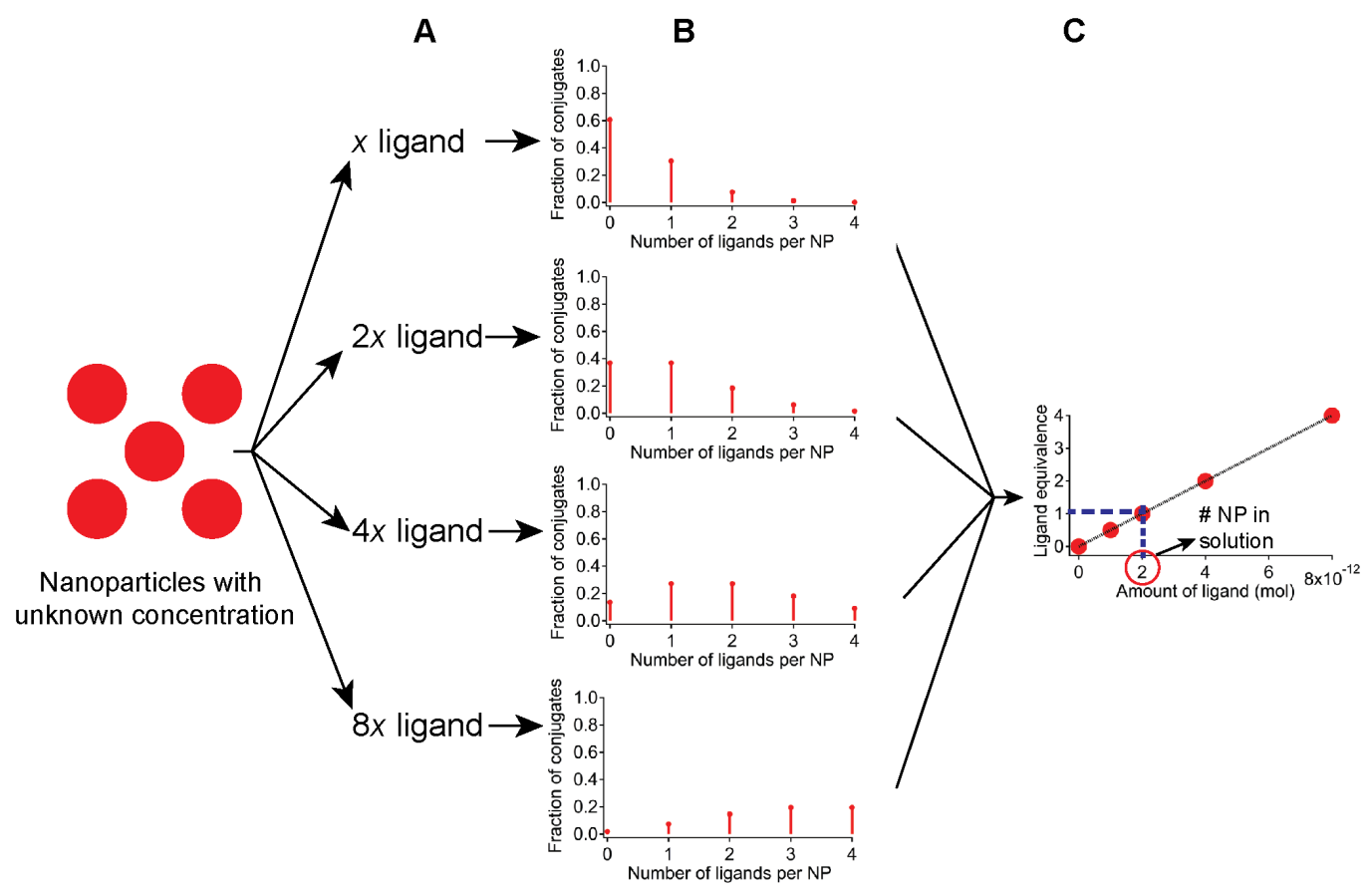

Figure 1. Schematic illustration for nanoparticle concentration determination from the distribution of nanoparticle-ligand conjugates. The steps of the protocol include: (A) Incubation of nanoparticles with a certain set of ligand quantities resulting in a statistical distribution of nanoparticle-ligand conjugates. (B) Identification and quantification of conjugates of varying valences using a suitable analytical technique. (C) Fitting the distribution of nanoparticle-ligand conjugates to a suitable statistical model provides the ligand equivalence as a function of quantity of ligand. The quantity of nanoparticles is equivalent to the quantity of ligand at a ligand equivalence of 1 .

The illustration in Figure 1 serves to outline the procedure used to determine nanoparticle concentration from the distribution of nanoparticle-ligand conjugates. When nanoparticles are incubated with defined equivalences of ligands, the high surface area of the nanoparticle results in the formation of a distribution of nanoparticle-ligand 
conjugates with varying valences. With suitable selection of the ligand, it is feasible to separate and quantify the conjugates of varying valences ${ }^{10-12}$. Under specific conditions, these distributions are known to conform to the Poisson model ${ }^{13,14}$, allowing the determination of ligand equivalence from the distribution of conjugates formed. The ability to model the nanoparticle-ligand distributions, combined with an accurate knowledge of the ligand concentration and reaction efficiency (or alternatively, a measure of the amount of ligand immobilized onto the nanoparticle), provides sufficient data for determination of the concentration of nanoparticle in solution.

There are numerous advantages offered by this method of concentration determination. While this approach relies on the availability of robust ligand conjugation strategies, ligand / biomolecule attachment onto nanoparticles is a prerequisite for nanoparticle use in analytical assays, and as such requires no additional experimentation to determine conjugation chemistries. Analyzing the distribution of nanoparticle-ligand conjugates does not depend on absolute signal intensities, but rather involves the relative quantification of conjugates of varying valences. Analogous to ratiometric methods of quantification, this serves to reduce variations that may be caused by instrumental fluctuations ${ }^{15}$. Lastly, the method is widely accessible as many well-established analytical techniques, such as single molecule fluorescence microscopy ${ }^{12}$ and separation modules such as electrophoresis ${ }^{10}$ and chromatography ${ }^{16}$, may be used to analyze the relative quantities of conjugates of varying valences.

In this work, the ability to predict nanoparticle concentration from the distribution of nanoparticle-ligand conjugates is investigated. Validation of this method was achieved using gold nanoparticles as a model system. The selection of gold nanoparticles was 
based on the availability of well established synthetic protocols and the ability to accurately determine gold nanoparticle concentration using UV-Vis spectrometry ${ }^{17}$. Gold nanoparticles, with a diameter of $5.9 \mathrm{~nm}$, were incubated with a polyethylene glycol based ligand at varying equivalences. The conjugates of varying valences were separated and quantified using agarose gel electrophoresis. The applicability of the Poisson model to predict the distribution of conjugates was validated for the gold nanoparticles. The nanoparticle concentration was obtained from the Poisson fitting method, and the results were compared with concentration determination by UV-Vis measurements. Further validation of the protocol was achieved using binary quantum dots $(\mathrm{CdSe} / \mathrm{ZnS}$; core/shell) as a different form of nanomaterial. The concentration of quantum dots obtained using the Poisson fitting method was compared to that obtained using UV-Vis analysis. A protocol was then developed to determine the concentration of nanoparticles in a solution with an unknown nanoparticle concentration. This protocol was applied towards the determination of the concentration and the extinction coefficient of alloyed quantum dots $\left(\mathrm{CdSe}_{x} \mathrm{~S}_{1-x} / \mathrm{ZnS}\right.$, core/shell) of three different emission wavelengths (525 $\mathrm{nm}, 575 \mathrm{~nm}$ and $630 \mathrm{~nm}$ ). These nanoparticles were selected because the optical emission wavelength is controlled by the composition of the core rather than the size of nanocrystal ${ }^{18}$, so that the location and breadth of the emission band is not uniquely representative of the nanoparticle size.

The outcome of these experiments is a reliable and accessible method for determination of nanoparticle concentration, which is a critical parameter when, for example, determining extinction coefficients, and when designing stoichiometric ratios to 
build decorated nanoparticles for applications such as for bioassays and chemicallyselective imaging.

\section{Materials and methods}

A detailed list of reagents and protocols is available in the Supporting Information.

\section{Gold nanoparticle functionalization with mPEG thiol}

Due to the propensity of thiols to form disulfides, the mPEG thiol $\left(M_{n} 6000 \mathrm{~g}\right.$ $\mathrm{mol}^{-1}$ ) was first incubated with a 10-fold molar excess of tris(2-carboxyethyl) phosphine in borate buffer (100 mM, pH 9.3) for 1 hour. Varying amounts of the mPEG thiol solution were then incubated with a fixed volume of the gold nanoparticle solution. Typically, $10 \mu \mathrm{L}$ of the gold nanoparticle solutions were used, and the concentrations of the mPEG thiol solutions were such that a maximum of only $10 \mu \mathrm{L}$ was required for the highest equivalence solutions. The mixtures were incubated at $50{ }^{\circ} \mathrm{C}$ in an oven for 2 hours.

Agarose gel electrophoretic analysis of gold nanoparticle - mPEG thiol conjugates.

$2 \%(\mathrm{w} / \mathrm{v})$ agarose gels were prepared using 0.5 X Tris-Borate EDTA $(44.5 \mathrm{mM}$ Tris, $44.5 \mathrm{mM}$ borate, $1 \mathrm{mM}$ EDTA, $\mathrm{pH} 8.3$ ) as the buffer. Prior to loading the samples onto the gel, the samples were mixed with $5 \mu \mathrm{L}$ of loading buffer (40\% (w/v) sucrose). The gel electrophoresis was run at a field strength of $5.7 \mathrm{~V} \mathrm{~cm}^{-1}$.

Imaging of the gel was done using a BioRad ChemiDoc XRS+ system. Image analysis was subsequently completed using ImageJ software, from which the intensity profile of each lane was obtained. The various peaks associated with the intensity profile were fit using a Gaussian function available in Igor Pro (WaveMetrics Inc, Portland, OR, USA). Quantification of the various peaks was achieved by integrating the area under 
each curve, and then this data was normalized to the total area of the electrochromatogram. Fitting the data to the Poisson function was also done using Igor Pro.

\section{Water-soluble quantum dots}

Quantum dots dissolved in organic solvents were made water-soluble using glutathione. Glutathione $(25 \mu \mathrm{mol})$ was dissolved in $100 \mu \mathrm{L}$ of tetramethylammonium hydroxide solution (25 wt. \% in methanol). This ligand solution was added to quantum dots $(0.5 \mathrm{mg})$ that were dissolved in $1 \mathrm{~mL}$ of chloroform.

After overnight incubation at room temperature, the QDs were pelleted by centrifugation and dissolved in $500 \mu \mathrm{L}$ of borate buffer. To remove excess ligand, the QDs were precipitated using sodium chloride and ethanol (2 x) and finally were resuspended in borate buffer.

\section{Quantum dot functionalization with mPEG hexahistidine.}

Dilutions of mPEG hexahistidine were prepared in borate buffer $(100 \mathrm{mM}, \mathrm{pH}$ 9.3). Glutathione coated quantum dots (typical volume was $10 \mu \mathrm{L}$ ) were incubated with varying amounts of mPEG hexahistidine in borate buffer at $50{ }^{\circ} \mathrm{C}$ for 2 hours. The dispersities of quantum dot-ligand conjugates were evaluated using agarose gel electrophoresis by a protocol similar to that described for the gold nanoparticles.

\section{Results and discussion}

\section{Synthesis and characterization of gold nanoparticles}

Gold nanoparticles (AuNPs) were synthesized by the reduction of aurochloric acid using tannic acid and citrate ${ }^{19}$. The nanoparticles were subsequently coated with bis( $p$-sulfonatophenyl)phenylphosphine (BSPP) to improve colloidal stability, and 
characterized using transmission electron microscopy (TEM) (Figure S1a) and UV-Vis spectrometry (Figure S1b). Based on the measurement of at least 50 particles from the TEM images, the gold nanoparticles had a mean diameter of $5.9 \pm 0.9 \mathrm{~nm}$. The concentration of the nanoparticles was obtained using the method outlined by Haiss et al

${ }^{17}$ (See Supporting Information, Equation S1). The concentration of the $5.9 \pm 0.9 \mathrm{~nm}$ nanoparticles was determined to be $1.5 \pm 0.4 \mu \mathrm{M}$. The uncertainty in the concentration of the nanoparticle is mainly attributed to the dispersion in the size of the nanoparticles.

\section{Statistical distribution of gold nanoparticle - ligand conjugates}

Quantifying the ligand distribution on nanoparticles requires a ligand-nanoparticle system that allows the separation and identification of the nanoparticle - ligand conjugates of varying valences. In this work, a thiol functionalized poly(ethylene glycol) methyl ether (mPEG thiol) was utilized as the ligand. Previous studies have demonstrated the use of poly(ethylene glycol) based ligands to modify nanoparticles, and the subsequent purification of nanoparticles with different valences ${ }^{10}$. Advantages of using poly(ethylene glycol) based ligands include commercial availability in a wide range of molecular weights and functionalities, and minimal non-specific interactions with nanoparticles. The thiol functionality of the mPEG ligand allows facile and strong immobilization onto the gold nanoparticles. 
(a)

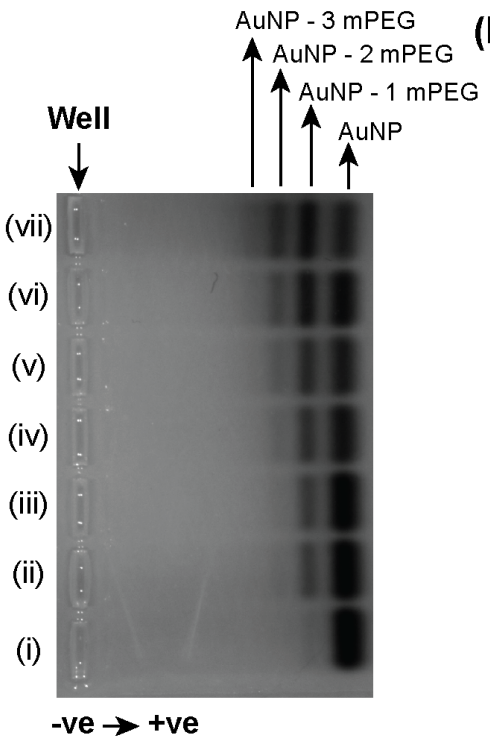

(b)

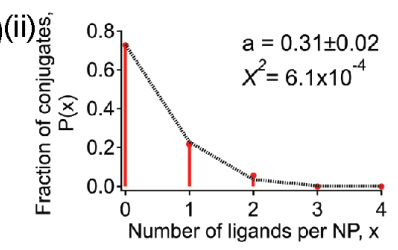

(iv)

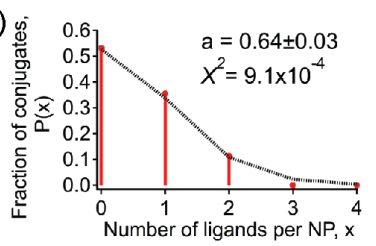

(vi)

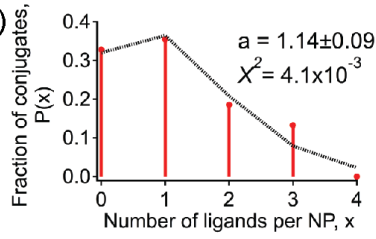

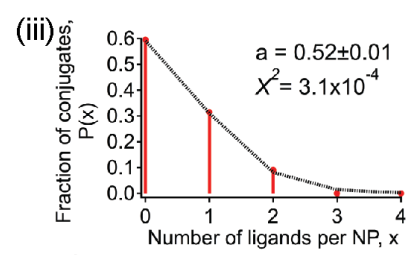

(v)
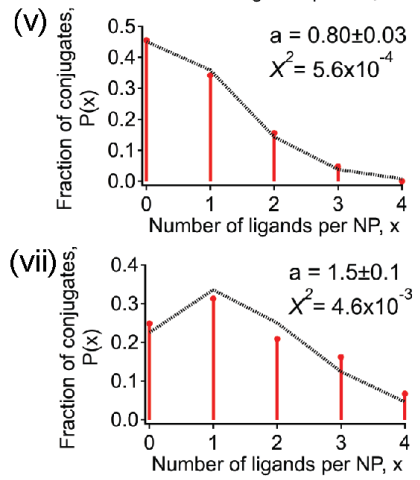

Figure 2. Statistical distribution of AuNP - mPEG thiol conjugates. (a) Agarose gel electrophoretic separation of AuNPs conjugated to varying amounts of mPEG thiol (mol) (i) 0 (ii) $3.2 \times 10^{-12}$ (iii) $6.3 \times 10^{-12}$ (iv) $9.5 \times 10^{-12}$ (v) $1.3 \times 10^{-11}$ (vi) $1.9 \times 10^{-11}$ (vii) $2.54 \times$ $10^{-11}$. The samples were run on a $2 \%$ agarose gel in $0.5 \times \mathrm{TBE}$ at a field strength of $5.7 \mathrm{~V}$ $\mathrm{cm}^{-1}$ (b) Comparing the distribution of AuNP - mPEG thiol conjugates (vertical lines with markers represent the density measurement associated with the gel from (a)) with the theoretical Poisson distribution (continuous lines). " $a$ " refers to the average ligand equivalence as calculated from the Poisson fit, and $X^{2}$ refers to the chi squared value used to determine the conformity of the fit. The mathematical relationship between $P(x), x$ and $a$ may be found in Equation 1. It should be noted that only Lane (vii) has a noticeable proportion of AuNP-4 mPEG conjugates, but may not be visibly discernable on the gel due to its low abundance and the low electrophoretic resolution between AuNP-3 mPEG and 4 mPEG conjugates. The analysis of the lane profile provides useful quantitative data

The BSPP coated gold nanoparticles (AuNPs) were incubated with varying amounts of mPEG thiol, with a highest equivalence ratio of two. The small equivalences that were used were intended to result in a clear distribution of conjugates with varying valences of AuNP-ligand conjugates. These conjugates were separated and quantified using agarose gel electrophoresis (Figure 2). In the agarose gel, the AuNPs had the highest electrophoretic mobility, with the mPEG thiol functionalized AuNPs having a progressively lower electrophoretic mobility as a function of the number of ligands immobilized on the particle. It was important to use a high molecular weight polymer (> 
$5000 \mathrm{~g} \mathrm{~mol}^{-1}$ ) to induce sufficient change in electrophoretic mobility for resolution using agarose gel electrophoresis ${ }^{10}$.

Quantitative analysis of the agarose gels provided data about the fraction of AuNP-mPEG thiol conjugates of different valences that formed when the nanoparticles were incubated with varying amounts of ligand. In Figure $2 b$, the distribution of conjugates is compared to that predicted by the Poisson distribution (Equation 1)

$$
P(x)=\frac{\left(e^{-a}\right)\left(a^{x}\right)}{x !} \quad \text { Equation } 1
$$

where $x$ refers to the number of ligands per particle, $P(x)$ refers to the fraction of conjugates with a ligand valency of $x$, and $a$ refers to the average number of ligands per nanoparticle.

Evidence for the conformity of nanoparticle-ligand distribution to the Poisson model is observed in Figure 2b. A qualitative comparison between the experimental data and the predictions of the Poisson function is possible from Figure $2 \mathrm{~b}$. The experimental data (vertical lines with markers) closely follows the Poisson model (continuous line), with only slight deviations observed for higher equivalences ( $\geq 1)$. The quality of fit can also be quantitatively judged from the chi squared $\left(X^{2}\right)$ value that is calculated when the information obtained from the gel electrophoresis analysis is fit to the Poisson model. A $X^{2}$ closer to zero indicates a better fit, and it is observed that equivalences greater than 1 have a $X^{2}$ value that is an order of magnitude greater than that at the lower equivalence. The greater deviations at the higher equivalences could be a consequence of the quantitative analysis of the gel electrophoresis images. The resolution of bands associated with the electrophoretic separation between conjugates of higher valences $(>3)$ is limited, 
and this reduces the accuracy associated with quantification. At higher equivalences, the fraction of conjugates with a valence greater than three becomes significant.

Although a number of mathematical functions have been proposed to model ligand distribution, the Poisson model is one of the simplest to predict nanoparticle-ligand distributions ${ }^{14}$. The calculation benefits from having only one unknown variable (equivalence of ligand per nanoparticle, $a$ ).

Certain assumptions need to be valid for the Poisson model to apply. First, the number of reactive sites must remain constant during the ligand modification process. This is generally applicable to nanoparticles, especially when incubated with small equivalents of ligand, as the large surface area of the nanoparticles can accommodate many more ligands. Rahme et al ${ }^{20}$ have quantified the amount of PEG that may be immobilized onto gold nanoparticles, and PEG with a molecular weight of $5400 \mathrm{~g} \mathrm{~mol}^{-1}$ was found to have a grafting density of $2.4 \mathrm{mPEG}$ molecules $\mathrm{nm}^{-2}$ on a $15 \mathrm{~nm}$ gold nanoparticle. The $5.9 \mathrm{~nm}$ gold nanoparticles have a surface area of approximately 109 $\mathrm{nm}^{2}$, which can accommodate 262 molecules of $\mathrm{mPEG}$ ligand. This is a large excess capacity when considering the maximum valence of $4 \mathrm{mPEG}$ ligands per nanoparticle obtained in the experiments described herein.

Second, binding events must be independent of each other. This is of concern when the immobilization of one ligand interferes with or templates subsequent ligand immobilization, either through steric or electrostatic interactions. The use of a neutral mPEG ligand eliminates electrostatic repulsion as a concern. It is assumed that the steric influences of the mPEG ligand on subsequent mPEG ligand chemisorption may be 
ignored at the low equivalences of ligand per nanoparticle that are used in these experiments $^{20}$.

Finally, the reaction between the ligand and nanoparticle should be irreversible. The thiol-gold bond is known to be thermodynamically stable ${ }^{21}$, and the reactions are performed at high nanoparticle concentrations $(\geq 0.5 \mu \mathrm{M})$, ensuring a low probability for the dissociation of ligands from the nanoparticle.

\section{Gold nanoparticle concentration determination using Poisson fitting}

Correlating the amount of ligand added with the distribution of nanoparticleligand conjugates requires accurate knowledge of ligand concentration and control of the conditions that ensure quantitative reaction of the ligand with the nanoparticles. The quantification of polymeric ligands is not trivial due to the distribution in their molecular weights. To obtain a more accurate measure of ligand concentration in solution, the thiol functionality of the mPEG ligand was quantified using 2-thiopyridone as a chromophoric label (See supporting information, Figure S2). By measuring the moles of thiol per gram of polymer, a corrected molecular weight of $7.02( \pm 0.06) \times 10^{3} \mathrm{~g} \mathrm{~mol}^{-1}$ was obtained for the thiol functionalized mPEG polymer. This corrected molecular weight was used to determine the amount of mPEG thiol that was incubated with the gold nanoparticles.

The reaction between the mPEG ligands and the AuNPs was carried out at $50{ }^{\circ} \mathrm{C}$ to ensure a quantitative reaction. The importance of heating the mixture is evident from the correlation curves depicted in Figure S3. The correlation curve serves to compare the equivalence of ligand per gold nanoparticle. This was evaluated by fitting the distribution of conjugates to the Poisson model and considering the actual quantity of ligand incubated with the gold nanoparticles. At room temperature (approx. $25^{\circ} \mathrm{C}$ ), a linear 
correlation was observed, but the slope of the line of best fit was less than 1 . The correlation curve for samples incubated at a higher temperature $\left(50{ }^{\circ} \mathrm{C}\right)$ was linear and had a slope of 0.97 , indicating high correlation between the amount of ligand incubated with the nanoparticles and the distribution of conjugates predicted by the Poisson function. The increased extent of reaction at the higher temperature may arise for two reasons. First, the immobilization of the mPEG thiol onto the gold nanoparticle would require the displacement of the phosphine ligand on the nanoparticle. Ligand exchange reactions typically require an excess of ligand as it is a mass-transfer dominated process. For the small equivalents of ligands used here, a higher temperature would favor the stronger thiol-gold bond over the phosphine-gold interaction ${ }^{22}$. Secondly, the thiol moiety of the mPEG ligand may be sterically blocked by the globular structure of the mPEG ligand. Using a higher temperature would facilitate the reaction between the mPEG ligand and the gold nanoparticle surface. It should be noted that when quantitative reactions are not possible, it is important to determine the total amount of ligand immobilized onto the nanoparticles, rather than use the quantity of ligand incubated with the nanoparticles. ${ }^{16}$
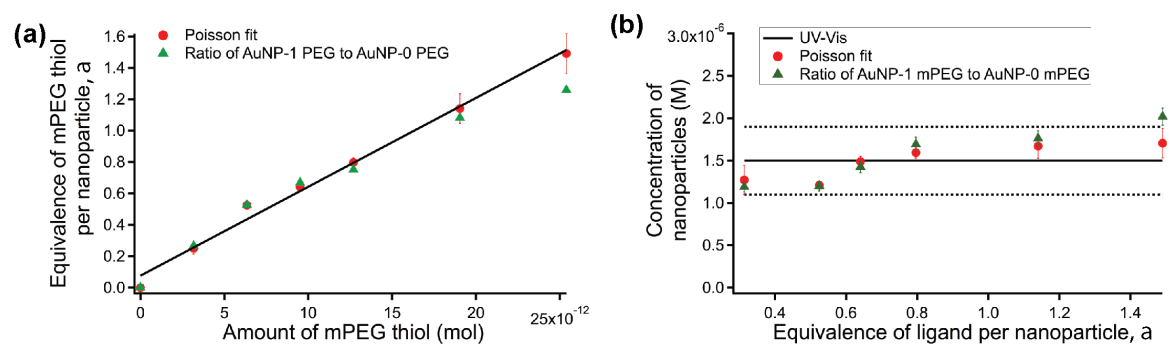

Figure 3. Determination of nanoparticle concentration from ligand distribution (a) Calibration curve illustrating the calculated ligand equivalence as a function of the amount of ligand added. The y-axis represents the ligand equivalence obtained by fitting the distribution of conjugates to the Poisson model (" $a$ " in Equation 1). Also plotted is the ratio of intensities for conjugates with one ligand and conjugates with no ligand (b) Correlation of the concentration determined by fitting the conjugate distribution to the 
Poisson model with the values determined by UV-Vis absorption spectroscopy for a range of values of equivalence of ligand per nanoparticle. All error bars represent the uncertainty (one standard deviation) in the prediction of ligand equivalence by the Poisson model.

Figure 3 presents the calibration curve that was constructed based on the data derived from the agarose gel and the known quantity of mPEG thiol ligand that was added for reactions. A linear correlation was obtained, with a correlation coefficient $\left(\mathrm{R}^{2}\right)$ of 0.99 , indicating the independence of reaction efficiency on the concentration of ligand for the ligand equivalences that were investigated. Using the line of best fit for the calibration curve, the concentration of the nanoparticles was determined to be $1.6 \pm 0.3$ $\mu \mathrm{M}$. This value was derived from the equation of the straight line by setting the equivalence of mPEG thiol per nanoparticle to one. At this equivalence, the moles of ligand are equal to the amount of nanoparticles in solution. Using the $t$-test at the $95 \%$ confidence interval, the concentration measured using the Poisson distribution was found to be the same as that measured using UV-Vis analysis (Table 1).

Figure $3 \mathrm{~b}$ illustrates the concentration of nanoparticle calculated at each individual point on the calibration curve illustrated in Figure 3a, and the data is placed in context by showing the concentration determined using UV-Vis analysis and its associated variance (due to the unavoidable distribution in nanoparticle size). At all the tested equivalences, the concentration measured at the individual points fall within the range of concentrations measured with UV-Vis analysis. It was observed that the concentration of AuNP measured at certain equivalences had deviations of up to 19 $\%$. This high deviation is observed for the lower ligand equivalences. At these ligand equivalences, the lower proportion of AuNP-2 mPEG conjugates makes it difficult to completely resolve bands associated with conjugates containing 1 and 2 mPEG 
ligands. This causes an overestimation in the proportion of mPEG 1 conjugates, which results in predicting higher ligand equivalences. This would result in the underestimation of nanoparticle concentration at low ligand equivalences, as is observed in Figure 3b. While using single point measurements to calculate nanoparticle concentration may not be accurate, taking the average of all the points provides a value that is highly consistent with that measured using UV-Vis (Table 1). Thus, the concentration determined from a single point should only serve as an estimate, with the average determined from at least five points (spanning ligand equivalences of 0.1 - 2) providing a more accurate value for nanoparticle concentration.

Also included in Figure 3a is data obtained by taking the ratio of the peak intensities for conjugates with one ligand (AuNP - $1 \mathrm{mPEG)}$ and nanoparticles without any attached ligand (AuNP $-0 \mathrm{mPEG)}$. The overlap between this ratio and the equivalences predicted by the Poisson model is significant, especially for equivalences less than 1. This provides a facile method for determining the concentration of the nanoparticles, as it does not require the quantification of conjugates with higher valences. The low conjugate valences are those that are the most clearly resolved in electrophoretic and chromatographic techniques ${ }^{11}$. Figure $3 b$ further indicates the similarity between the concentration determined using such a ratiometric method and that predicted by UV-Vis analysis. The ratiometric method of determining the concentration of nanoparticles is only applicable for equivalences less than one. For equivalences greater than one, fitting the band intensities to the Poisson model provides for a more accurate estimation of nanoparticle concentration. 
Table 1 Summary of nanoparticle concentrations measured using UV-Vis and by fitting conjugate distributions to the Poisson model. For the Poisson model method, three different mathematical treatments of the data were used for concentration determination, including use of a linear calibration curve, individual data points and a ratiometric approach.

\begin{tabular}{|c|c|}
\hline Method for concentration determination & Concentration of gold nanoparticles ( $\boldsymbol{\mu M})$ \\
\hline UV-Vis spectroscopy & $1.5 \pm 0.4$ \\
\hline Linear fit of Poisson model & $1.6 \pm 0.3$ \\
\hline Average of individual points of Poisson fit & $1.4 \pm 0.3$ \\
\hline Ratio of AuNP- 1 mPEG to AuNP - 0 mPEG & $1.5 \pm 0.4$ \\
\hline
\end{tabular}

The precision of the assay would be defined by the reproducibility of the distribution of nanoparticle-ligand conjugates that are formed at various equivalences of ligand. The precision of the assay was evaluated by incubating multiple aliquots of the $5.9 \mathrm{~nm}$ AuNP sample with a specific equivalence of the mPEG thiol ligand. Agarose gel electrophoresis was subsequently used to analyze the nanoparticle - ligand distribution (Figure S4) and this was performed for a total of three different ligand equivalences. Fitting the data to the Poisson model provided equivalence values of $0.76 \pm 0.05,0.82 \pm$ 0.04 and $1.01 \pm 0.07$ for the three different gels depicted in Figure S4. The coefficient of variation ranged between $5-7 \%$ for the three different equivalences tested, indicating minimal variations between different samples. It should be noted that the uncertainty in the predicted ligand equivalence (as per the Poisson model) was greater than the variations between replicate samples. Thus, the standard error that is reported in all calibration curves represents the uncertainty in the predication of the nanoparticle-ligand equivalence by the Poisson model.

To further evaluate the repeatability of the method, the concentration of two samples of gold nanoparticles (from a single batch) were determined using the Poisson method on two different days. The concentrations of nanoparticles derived from the two 
sets of experiments are $1.5 \pm 0.2 \mu \mathrm{M}$ and $1.6 \pm 0.2 \mu \mathrm{M}$. These two values are the same statistically when compared by using the $t$-test at the $95 \%$ confidence interval.

Further more, the method was also validated by the use of a thiol functionalized nucleic acid as the ligand. Nucleic acids are common ligands used for the modification of gold nanoparticles ${ }^{23,24}$. The experimental details and subsequent data analysis can be found in the Supporting information. Applying the Poisson fitting method (described above) to the quantitative data obtained from the electrophoretic analysis of AuNP-DNA conjugates provided the nanoparticle concentration. The nanoparticle concentration was measured to be $2.7 \pm 0.1 \mu \mathrm{M}$, which deviated by only $4 \%$ from the actual concentration $(2.8 \pm 0.2 \mu \mathrm{M})$, as measured using UV-Vis analysis.

\section{Determination of nanoparticle concentration in an unknown solution}

From the above discussion, a ligand equivalence of less than or equal to 2 is ideal for the determination of nanoparticle concentration. For these ligand equivalences, the nanoparticle - ligand conjugates of varying valences are accurately identified, as the first band is always nanoparticles with no ligand. In addition, at higher ligand equivalences, the proportion of conjugates with a ligand valence greater than three is higher, and these conjugates are harder to resolve and accurately quantify.

For a solution of nanoparticles with unknown concentration, incubating a fixed volume of nanoparticle with a series of ligand quantities and analyzing the distribution of nanoparticle - ligand conjugates should provide sufficient data to estimate the nanoparticle concentration. The choice of ligand quantities must ensure that they are between 0.1 and 2 equivalents with respect to the potential nanoparticle concentration. For example, for a $10 \mu \mathrm{L}$ solution of nanoparticles that is assumed to be between $1 \mu \mathrm{M}$ 
and $1 \mathrm{mM}$ (3 orders of magnitude), a series of only 8 ligand amounts is required to elucidate the nanoparticle concentration. The ligand amounts range from $1 \times 10^{-11} \mathrm{~mol}$ to $1 \times 10^{-9} \mathrm{~mol}$, with all ligand amounts in between differing by a factor of two. This ensures that any concentration of nanoparticle in the above-defined range has a particular ligand amount that is within 0.1 to 2 equivalents, as is required to estimate the nanoparticle concentration. A demonstration of this principle is shown for a sample of $5.9 \mathrm{~nm}$ gold nanoparticles (Figure 4).
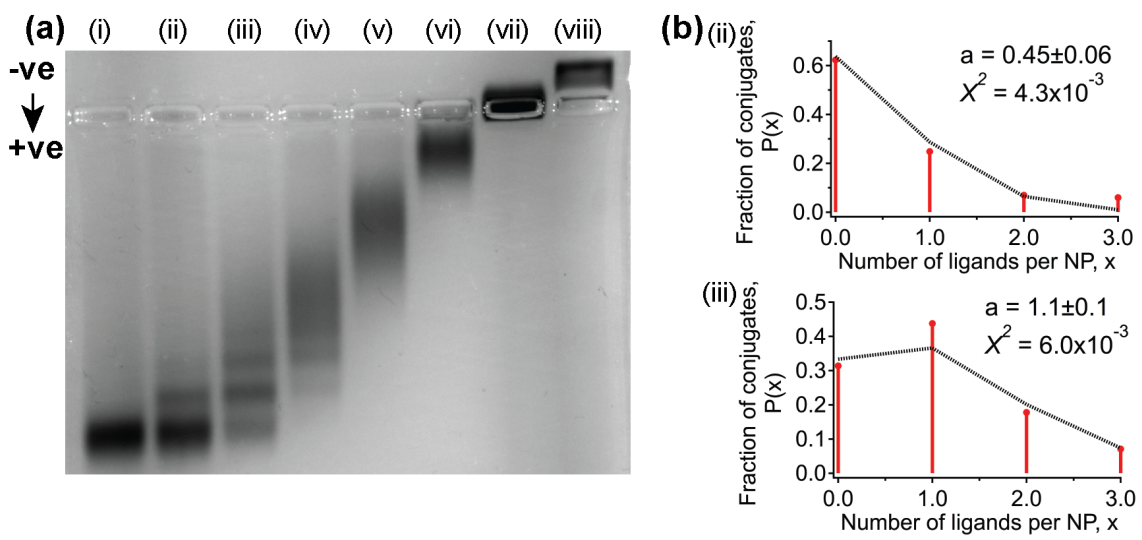

Figure 4. Determination of nanoparticle concentration in an unknown sample with an assumed concentration between $1 \mu \mathrm{M}$ and $1 \mathrm{mM}$ (a) Agarose gel electrophoretic analysis of gold nanoparticles $(10 \mu \mathrm{L})$ incubated with varying amounts of mPEG thiol (mol) (i) 0 (ii) $0.013 \times 10^{-9}$ (iii) $0.027 \times 10^{-9}$ (iv) $0.054 \times 10^{-9}$ (v) $0.107 \times 10^{-9}$ (vi) $0.214 \times 10^{-9}$ (vii) $0.429 \times 10^{-9}$ (viii) $0.857 \times 10^{-9}$. The samples were run on a $2 \%$ agarose gel in $0.5 \times \mathrm{TBE}$ at a field strength of $5.7 \mathrm{~V} \mathrm{~cm}^{-1}$. (b) Comparison between the distribution of conjugates measured from the agarose gel electrophoretic analysis and the distribution predicted by the Poisson model. The low $X^{2}$ value obtained for both fits indicates the applicability of the Poisson model to the distribution of nanoparticle-ligand conjugates. The mathematical relationship between $P(x), x$ and $a$ is found in Equation 1 .

The agarose gel image in Figure 4a represents $5.9 \mathrm{~nm}$ gold nanoparticles that were incubated with varying amounts of ligand. Lanes (ii) and (iii) provide distributions that may be quantified and fit to the Poisson model, and these fits are included in Figure $4 \mathrm{~b}$. The concentration determined from these two fits was $2.8 \pm 0.5 \mu \mathrm{M}$, which is similar to the concentration obtained by UV-Vis analysis $(3.1 \pm 0.7 \mu \mathrm{M})$. The values of 
concentration determined by the Poisson fit method and by UV-Vis are statistically the same as determined by a t-test at the $95 \%$ confidence interval. As previously discussed, increasing the number of points from which the nanoparticle concentration is determined would improve the accuracy of the measurement.

\section{Concentration determination of binary quantum dots (CdSe/ZnS; core/shell)}

Further validation of the Poisson fitting method for nanoparticle concentration determination was obtained when applied to semiconductor nanocrystals $(\mathrm{CdSe} / \mathrm{ZnS}$, core/shell), also referred to as quantum dots (QDs). These nanoparticles were made water-soluble using glutathione as a surface capping molecule. A sample of these nanoparticles was analyzed using a UV-Vis absorbance spectrometer (See Figure S6 for the UV-Vis spectra). The concentration of the QDs was determined from the absorbance spectrum using the method proposed by $\mathrm{Yu}$ et $\mathrm{al}^{4}$. The concentration of nanoparticles was determined to be $9.1 \pm 0.9 \mu \mathrm{M}$.

To use the Poisson method for the determination of QD concentrations, hexahistidine functionalized mPEG ligands (mPEG hexahistidine) were immobilized onto the glutathione functionalized quantum dots (GSH-QDs) in varying ratios, followed by determination of the distribution of conjugates that were formed. It was necessary to use a hexahistidine functionalized mPEG rather than the thiol functionalized MPEG to obtain quantitative reaction between the ligand and nanoparticle. Although thiol functional groups may also interact with the zinc on the QD surface, low reaction efficiencies were observed when the GSH QDs were incubated with mPEG thiol ligands (data not shown). This may be attributed to the bidentate binding capability of the GSH ligand ${ }^{25}$, which greatly reduced mono-thiol ligand displacement of the GSH, especially 
for the low equivalences $(<2)$ of ligand used. The concentration of mPEG hexahistidine was determined using the procedure outlined by Sims et al. ${ }^{26}$ (Supporting information S7). It was also observed that incubation at a higher temperature $\left(50^{\circ} \mathrm{C}\right)$ was required to achieve quantitative reaction between the hexahistidine mPEG ligand and the QD.
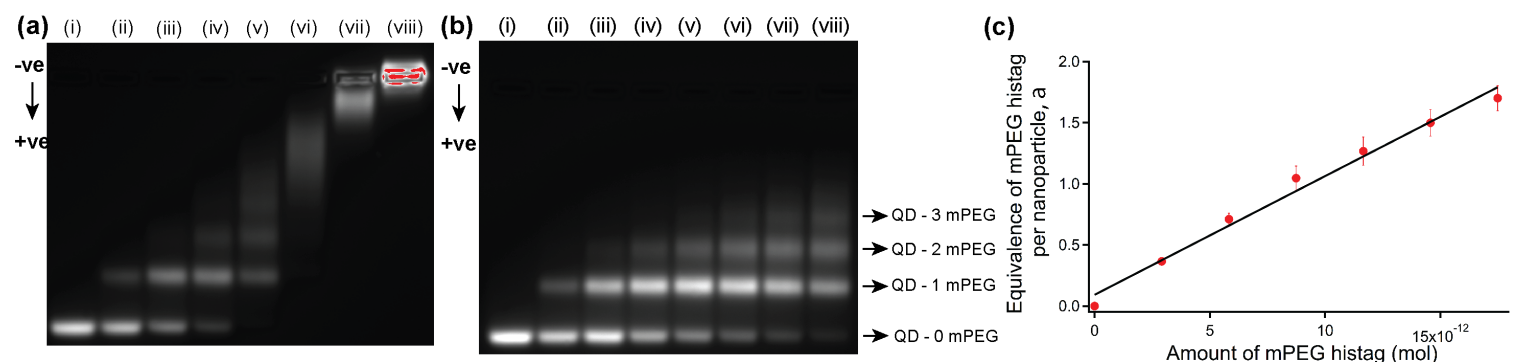

Figure 5. Concentration determination of $\mathrm{CdSe} / \mathrm{ZnS}$ (core/shell) quantum dots using the Poisson fitting method. (a) Agarose gel electrophoretic analysis of QDs incubated with varying amounts of ligand (mol) to provide an initial estimate of nanoparticle concentration. (i) 0 (ii) $2.92 \times 10^{-12}$ (iii) $5.84 \times 10^{-12}$ (iv) $1.17 \times 10^{-11}$ (v) $2.34 \times 10^{-11}$ (vi) $4.67 \times 10^{-11}$ (vii) $9.34 \times 10^{-11}$ (viii) $1.87 \times 10^{-10}$. (b) Agarose gel electrophoresis of QDs incubated with finer increments of mPEG hexahistidine (mol) (i) 0 (ii) $2.91 \times 10^{-12}$ (iii) $5.83 \times 10^{-12}$ (iv) $8.74 \times 10^{-12}$ (v) $1.17 \times 10^{-11}$ (vi) $1.46 \times 10^{-11}$ (vii) $1.75 \times 10^{-11}$ (viii) $2.33 \times$ $10^{-11}$. The samples were run on a $2 \%$ agarose gel in $0.5 \times \mathrm{TBE}$ at a field strength of $5.7 \mathrm{~V}$ $\mathrm{cm}^{-1}$. (c) Calibration curve illustrating the dependence of the ligand equivalence per nanoparticle (as determined from the Poisson distribution, " $a$ " in Equation 1) to the amount of mPEG hexahistidine added. The error bars (one standard deviation) represent the uncertainty in the prediction of the ligand equivalence by the Poisson model.

Following the protocol developed using gold nanoparticles, the GSH QDs were initially assumed to be within $1 \mu \mathrm{M}$ and $1 \mathrm{mM}$, and were incubated with specific amounts of ligand as previously described. Analysis of the conjugates was done using agarose gel electrophoresis (Figure 5a). Identity of the conjugates was assigned based on the fact that the GSH QDs with no mPEG ligand had the greatest mobility and the QDs modified with mPEG hexahistidine had a progressively lower electrophoretic mobility as a function of the number of ligands that were immobilized. The distribution of the conjugates was determined from lanes (i), (ii) and (iii) of Figure 5a and the distribution was fit to the Poisson model. The resulting equivalences were used to estimate the nanoparticle 
concentration. The concentration of the GSH QDs was determined to be $8 \pm 1 \mu \mathrm{M}$, which suggests a deviation in accuracy of about $10 \%$ when compared to the value of $9.1 \pm 0.9$ $\mu \mathrm{M}$ that was determined using UV-Vis. Having identified the correct range of concentration, a more rigorous analysis was conducted to improve the accuracy. The GSH QDs were incubated with finer increments over a narrower range of mPEG hexahistidine, and the resulting conjugates were again analyzed using agarose gel electrophoresis (Figure 5b). Fitting the distribution of conjugates to the Poisson model provided the equivalence ratios that are used to construct the plot in Figure 5c. Using the equation of the line of best fit provided a value of $9.3 \pm 0.7 \mu \mathrm{M}$, which is only $2 \%$ different from the concentration of $9.1 \pm 0.9 \mu \mathrm{M}$ determined by the UV-Vis method.

\section{Concentration determination of alloyed semiconductor quantum dots}

The method was applied to provide concentration data for the subsequent determination of the extinction coefficient of alloyed semiconductor nanoparticles. Alloyed quantum dots $\left(\mathrm{CdS}_{\mathrm{x}} \mathrm{Se}_{1-\mathrm{x}} / \mathrm{ZnS}\right.$ core/shell; QD) of three different emission wavelengths $(525 \mathrm{~nm}, 575 \mathrm{~nm}$ and $630 \mathrm{~nm})$ were obtained from a commercial source. They were made water-soluble by replacing the original oleic acid coating with glutathione. The core of these nanoparticles consists of a mixed composition of cadmium, selenium and sulfur, prohibiting the use of equations and models previously used for the binary QDs (core composition of only $\mathrm{CdSe}$ ) to determine nanoparticle concentration from a UV-Vis spectrum. 


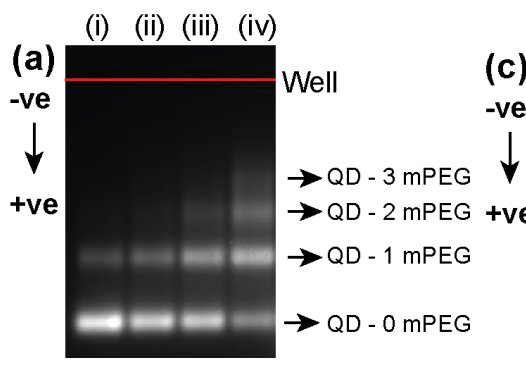

(b)

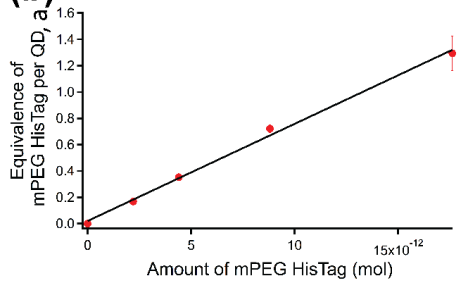

(i) (ii) (iii) (iv)

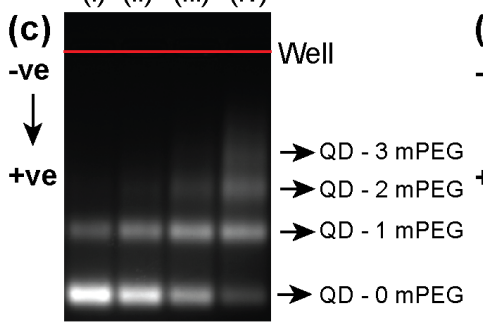

(d)

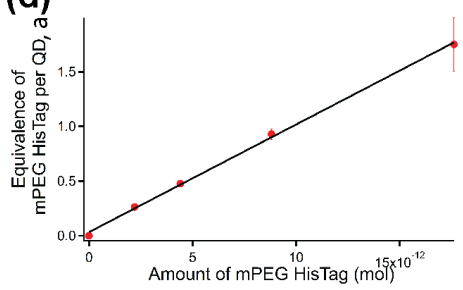

(i) (ii) (iii) (iv)

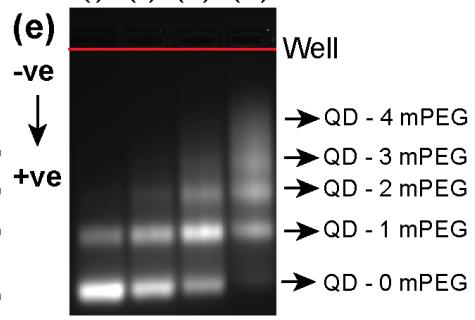

(f)

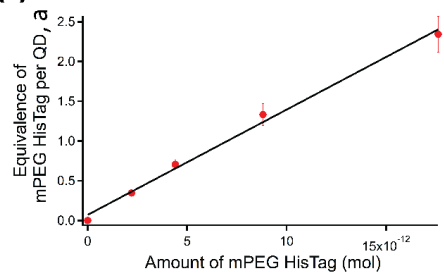

Figure 6. Distribution of ligand nanoparticle conjugates for QD525, QD575 and QD630 functionalized with varying equivalents of $\mathrm{mPEG}$ hexahistidine. Agarose gel electrophoretic analysis of QDs (a: QD525, c: QD575 and e: QD630) incubated with varying amounts of hexahistidine mPEG ligand (i) $1.76 \times 10^{-12} \mathrm{~mol}$ (ii) $3.52 \times 10^{-12} \mathrm{~mol}$ (iii) $7.05 \times 10^{-12} \mathrm{~mol}$ (iv) $1.41 \times 10^{-11} \mathrm{~mol}$. (b), (d) and (f) Calibration curves illustrating the calculated ligand equivalence as a function of the amount of ligand added for the different QDs. The y-axis represents the ligand equivalence obtained by fitting the distribution of conjugates to the Poisson model (" $a$ " in Equation 1). The samples were run on a $2 \%$ agarose gel in $0.5 \mathrm{x}$ TBE at a field strength of $5.7 \mathrm{~V} \mathrm{~cm}^{-1}$. The error bars represent one standard deviation, and are a measure of the uncertainty in the prediction of the ligand equivalence by the Poisson model.

Figure 6 illustrates the electrophoretic analysis of the alloyed GSH QDs that were incubated with varying amounts of ligand. The ligand amounts were chosen based on the assumption that the concentration of the nanoparticles was within 1 and $20 \mu \mathrm{M}$. The high negative charge density of the GSH coating caused the GSH QDs to move towards the positive electrode under the influence of the applied electric field. Addition of mPEG ligands onto the QDs resulted in a decrease in electrophoretic mobility. These observations are similar to those obtained for the binary QDs (CdSe/ZnS, core/shell). Quantitative analysis of the distribution of conjugates from the gel electrophoretic images provided data that was fit to the Poisson model. These were plotted, along with the 
amounts of ligand, to provide the calibration curves in Figure $6 \mathrm{~b}, \mathrm{~d}$ and $\mathrm{f}$. The concentrations of the nanoparticles determined from these calibration curves, in conjunction with their absorbance spectra (Figure S8), enabled the determination of the extinction coefficient of the alloyed QDs (Table 2). To the best of the author's knowledge, these values represent one of the first estimations of the extinction coefficient of alloyed QDs $\left(\mathrm{CdS}_{\mathrm{x}} \mathrm{Se}_{1-\mathrm{x}} / \mathrm{ZnS}\right.$; core/shell $)$.

Table 2 Extinction coefficients of alloyed semiconductor nanocrystals of three different emission wavelengths as determined by the Poisson method.

\begin{tabular}{|c|c|c|}
\hline Quantum dot & $\begin{array}{c}\text { Wavelength of first exciton } \\
\text { peak (nm) }\end{array}$ & $\begin{array}{c}\text { Extinction coefficient at first } \\
\text { exciton peak }\left(\mathbf{M}^{\mathbf{- 1}} \mathbf{c m}^{\mathbf{- 1}}\right)\end{array}$ \\
\hline QD525 & 504 & $2.1 \pm 0.1 \times 10^{5}$ \\
\hline QD575 & 552 & $2.2 \pm 0.2 \times 10^{5}$ \\
\hline QD630 & 612 & $2.2 \pm 0.2 \times 10^{5}$ \\
\hline
\end{tabular}

Within experimental error, the extinction coefficients of QDs of the three different emission wavelengths were identical. Alloyed QDs have their emission wavelength tuned by the composition of the core, rather than its size ${ }^{18}$. They are expected to have similar extinction coefficients, as long as the size of the nanocrystal is kept consistent ${ }^{27}$.

To corroborate the accuracy of the results, the extinction coefficient of alloyed QD525 was determined using an alternative method. Previously, a protocol for the preparation and isolation of quantum dots functionalized by a single nucleic acid was proposed $^{28}$. Using this method, the QD525 was conjugated to a single nucleic acid strand that was co-labeled with a Cy5 dye. In a sample of monoconjugates, the amount of Cy5 is equal to the amount of QDs. UV-Vis analysis of these conjugates (Figure S9b) provides a method to determine the extinction coefficient of the QDs as the extinction coefficient of Cy5 is known to be $2.5 \times 10^{5} \mathrm{M}^{-1} \mathrm{~cm}^{-1}$. Based on this analysis, the extinction coefficient of 
QD525 was found to be $2.1( \pm 0.2) \times 10^{5} \mathrm{M}^{-1} \mathrm{~cm}^{-1}$, which is in agreement with the value obtained using the Poisson fitting method (Table 2).

\section{Conclusions}

A simple method using equipment that is widely available was developed for the determination of the concentration of a variety of different types of nanoparticles. The method exploits the quantitative relationships associated with the distribution of conjugates that are formed when nanoparticles are incubated with small $(<2)$ equivalents of ligand. Fitting such distributions to the Poisson model provided the equivalence of the ligand that was used. The equivalence in combination with knowledge of the amount of ligand allows the determination of nanoparticle concentration. The method was validated by determining the concentration of gold nanoparticles (5.9 $\mathrm{nm}$ diameter) and was found to correlate well with the concentrations determined using UV-Vis analysis. The concentrations determined using the Poisson fitting method differed by only $7 \%$ from that determined using UV-Vis analysis. The precision of the assay was defined by the reproducibility obtained in the statistical distribution of nanoparticle-ligand conjugates at the different ligand eqivalences. Based on replicate analysis, a coefficient of variation of 5-7 \% was obtained for three different ligand equivalences. The repeatability of the assay was also tested by measuring the concentration of a sample of gold nanoparticles on two different days and the two values obtained was found to be the same as determined by a ttest at $95 \%$ confidence. Based on the validation studies, a set of guidelines was developed to facilitate the determination of nanoparticle concentration in an unknown solution. Further validation was also obtained by measuring the concentration of binary 
quantum dots $(\mathrm{CdSe} / \mathrm{ZnS}$; core/shell), and was found to correlate well (2\% deviation) with the concentration of the quantum dots as determined using UV-Vis analysis.

The protocol was further applied for an accurate determination of the extinction coefficient of alloyed semiconductor nanoparticles $\left(\mathrm{CdSe}_{\mathrm{x}} \mathrm{S}_{1-\mathrm{x}} / \mathrm{ZnS}\right.$, core/shell) of three different emission wavelengths $(525 \mathrm{~nm}, 575 \mathrm{~nm}$ and $630 \mathrm{~nm})$. The QDs of the different emission wavelengths were found to have similar extinction coefficients $\left(\sim 2.1 \times 10^{5} \mathrm{M}^{-}\right.$

$\left.{ }^{1} \mathrm{~cm}^{-1}\right)$. This is a defining characteristic of these nanoparticles, and this helps to further validate the use of the Poisson method for nanoparticle concentration determination.

\section{Acknowledgements}

We would like to acknowledge the Natural Sciences and Engineering Research Council of Canada (NSERC) for financial support of this research and also for providing U.U. with a graduate fellowship. Dr. Ilya Gourevich from the Centre for Nanostructure Imaging at University of Toronto is acknowledged for assistance with obtaining the TEM images. Dr. Travis L. Jennings is acknowledged for the provision of a sample of quantum dots from eBiosciences.

\section{Associated Content}

Supporting information: Detailed experimental procedures, description of instruments used, characterization of the gold nanoparticles, quantification of mPEG thiol and mPEG hexahistidine, evaluation of assay precision, reaction temperature optimization, UV-Vis analysis of all quantum dots used and synthesis of monovalent QD-DNA conjugates. This material is available free of charge via the Internet at http://pubs.acs.org.

\section{References}

(1) Zamborini, F. P.; Bao, L.; Dasari, R. Anal. Chem. 2012, 84, 541-576. 
(2) Cho, E. J.; Holback, H.; Liu, K. C.; Abouelmagd, S. A.; Park, J.; Yeo, Y. Mol. Pharmaceutics 2013, 10, 2093-2110.

(3) López-Serrano, A.; Olivas, R. M.; Landaluze, J. S.; Cámara, C. Anal. Methods 2014, 6, 38-56.

(4) Yu, W. W.; Qu, L.; Guo, W.; Peng, X. Chem. Mater. 2003, 15, 2854-2860.

(5) Shang, J.; Gao, X. Chem. Soc. Rev. 2014, 43, 7267-7278.

(6) Dai, Q.; Wang, Y.; Li, X.; Zhang, Y.; Pellegrino, D. J.; Zhao, M.; Zou, B.; Seo, J.; Wang, Y.; Yu, W. W. ACS Nano 2009, 3, 1518-1524.

(7) Laborda, F.; Jiménez-Lamana, J.; Bolea, E.; Castillo, J. R. J. Anal. At. Spectrom. 2013, 28, 1220-1232.

(8) Li, M.; Guha, S.; Zangmeister, R.; Tarlov, M. J.; Zachariah, M. R. Langmuir 2011, 27, 14732-14739.

(9) Röding, M.; Deschout, H.; Braeckmans, K.; Rudemo, M. Phys. Rev. E 2011, 84, 031920.

(10) Sperling, R. A.; Pellegrino, T.; Li, J. K.; Chang, W. H.; Parak, W. J. Adv. Funct. Mater. 2006, 16, 943-948.

(11) Claridge, S. A.; Liang, H. W.; Basu, S. R.; Fréchet, J. M. J.; Alivisatos, A. P. Nano Lett. 2008, 8, 1202-1206.

(12) Casanova, D.; Giaume, D.; Moreau, M.; Martin, J.-L.; Gacoin, T.; Boilot, J.-P.; Alexandrou, A. J. Am. Chem. Soc. 2007, 129, 12592-12593.

(13) Mullen, D. G.; Banaszak Holl, M. M. Acc. Chem. Res. 2011, 44, 1135-1145.

(14) Hakem, I. F.; Leech, A. M.; Johnson, J. D.; Donahue, S. J.; Walker, J. P.; Bockstaller, M. R. J. Am. Chem. Soc. 2010, 132, 16593-16598.

(15) Noor, M. O.; Shahmuradyan, A.; Krull, U. J. Anal. Chem. 2012.

(16) Mullen, D. G.; Fang, M.; Desai, A.; Baker, J. R., Jr.; Orr, B. G.; Banaszak Holl, M. M. ACS Nano 2010, 4, 657-670.

(17) Haiss, W.; Thanh, N. T. K.; Aveyard, J.; Fernig, D. G. Anal. Chem. 2007, 79, 4215-4221.

(18) Bailey, R. E.; Nie, S. J. Am. Chem. Soc. 2003, 125, 7100-7106.

(19) JW, S.; HJ, G. Eur J Cell Biol 1985, 38, 87-93.

(20) Rahme, K.; Chen, L.; Hobbs, R. G.; Morris, M. A.; O'Driscoll, C.; Holmes, J. D. RSC Adv. 2013, 3, 6085 .

(21) Häkkinen, H. Nature Chem. 2012, 4, 443-455.

(22) Bain, C. D.; Evall, J.; Whitesides, G. M. J. Am. Chem. Soc. 1989, 111, 71557164.

(23) Parak, W. J.; Pellegrino, T.; Micheel, C. M.; Gerion, D.; Williams, S. C.; Alivisatos, A. P. Nano Lett. 2003, 3, 33-36.

(24) Pellegrino, T.; Sperling, R. A.; Alivisatos, A. P.; Parak, W. J. J. Biomed and Biotechnol. 2007, 2007, 1-9.

(25) Chan, W. C. W.; Prendergast, T. L.; Jain, M.; Nie, S. Bornhop, D. J.; Licha, K., Eds.; SPIE, 2000; Vol. 3924, pp. 2-9.

(26) Sims, G. E. C.; Snape, T. J. Anal. Biochem. 1980, 107, 60-63.

(27) Cytodiagnostics Inc.

(28) Uddayasankar, U.; Zhang, Z.; Shergill, R. T.; Gradinaru, C. C.; Krull, U. J. Bioconjugate Chem. 2014, 25, 1342-1350. 


\title{
The evaluation of nanoparticle-ligand distributions to determine nanoparticle concentration
}

\author{
Uvaraj Uddayasankar, Ravi T. Shergill and Ulrich J. Krull* \\ Department of Chemical and Physical Sciences, University of Toronto Mississauga, 3359 \\ Mississauga Road, Mississauga, ON, Canada L5L 1C6 \\ *E-mail: ulrich.krull@utoronto.ca, Fax number: 9055694388
}

\section{TABLE OF CONTENTS}

1. Materials S-2

2. Instrumentation..................................... -2

3. Synthesis and characterization of gold nanoparticles..............S-4

4. Determination of gold nanoparticle concentration using UV-Vis analysis................................... S-5

5. Quantification of mPEG thiol ............................ S-5

6. Influence of reaction temperature on the extent of reaction between gold nanoparticles and mPEG thiol........... S-6

7. Evaluation of the precision in the distribution of conjugates....... S-7

8. AuNP-DNA conjugate distributions to determine concentration ... S-9

9. UV-Vis analysis of binary QDs (CdSe/ZnS; core/shell)............ S-9

10. Hexahistidine functionalized $\mathrm{mPEG} \ldots \ldots \ldots \ldots \ldots \ldots \ldots \ldots \ldots \ldots . . \ldots \ldots$

11. UV-Vis analysis of alloyed semiconductor nanocrystals.......... S-11

12. Synthesis of monovalent QD-DNA conjugates................. S-11 


\section{Materials}

Gold (III) chloride trihydrate, tannic acid, trisodium citrate dihydrate, bis(psulfonatophenyl)phenyl phosphine, sodium borohydride, poly(ethylene glycol) methyl ether thiol $\left(M_{n} 6000\right)$, tris(2-carboxylethyl)phosphine hydrochloride, Aldrithiol-2, barium chloride, iodine, glutathione, tetramethylammonium hydroxide solution $(25$ wt. \% in methanol) and agarose were obtained from Sigma-Aldrich (Oakville, ON, Canada). All organic solvents were obtained from Caledon labs (Georgetown, ON, Canada). illustra NAP-5 desalting columns and His GraviTrap columns were purchased from GE Life Sciences (Quebec, Canada).

$\mathrm{CdS}_{\mathrm{x}} \mathrm{Se}_{1-\mathrm{x}} / \mathrm{ZnS}$ (core/shell) alloyed semiconductor nanoparticles with emission wavelengths of $525 \mathrm{~nm}, 575 \mathrm{~nm}$ and $630 \mathrm{~nm}$ was obtained from Cytodiagnostics Inc. (Burlington, ON, Canada). CdSe/ZnS (core/shell) quantum dots, with an emission at 605 nm, were obtained from eBiosciences (San Diego, CA, USA)

Peptide sequences were obtained from CanPeptide Inc (Montreal, QC, Canada):

(6-Maleimidohexanoic acid) - G(Aib)GHHHHHH

DNA sequences were obtained from IDT DNA (Coraville, IA, USA):

5'-TTT TTT TTT TCC GAG CCT TAC TTC CAT GAT TTC TTT AAC TGC TCG GTT TTT TTT TT - Thiol - 3'

\section{Buffers:}

Borate buffer: $100 \mathrm{mM}$ borate, $\mathrm{pH} 9.25$

1 x TBE: 89 mM Tris, $89 \mathrm{mM}$ boric acid, 2 mM ethylenediaminetetraacetic acid. $\mathrm{pH} 8.3$

\section{Instrumentation}


UV-Visible spectra was obtained on a HP8452A diode-array spectrophotometer (Hewlett Packard Corporation, Pao Alto, CA, USA). The agarose gels were analyzed using a BioRad ChemiDoc XRS+ (Bio-Rad, Hercules, CA, USA). SEM images were obtained using a Hitachi S-5200 SEM (Hitachi High Technologies America, Pleasanton, CA, USA).

\section{Data analysis}

All data analysis was performed using Igor Pro (Ver. 6.3; WaveMetrics, Portland, OR). When the data was fit to the Poisson model, the minimization of the $X^{2}$ was used to determine the best fit ${ }^{1}$. The following equation is used by the software to calculate $X^{2}$,

$$
\chi^{2}=\sum_{i} \frac{\left(y-y_{i}\right)}{\sigma_{i}}
$$

where $y$ is the expected value for a given point, $y_{\mathrm{i}}$ is the measured value for that point and $\sigma_{\mathrm{i}}$ is the standard deviation associated with $\mathrm{y}_{\mathrm{i}}$.

To compare the mean values obtained by different methods, the t-test was used. The t-test is a statistical test used to quantify the difference between two mean values ${ }^{2}$. The t-statistic was calculated using the following equation,

$$
t=\frac{x_{1}-x_{2}}{\sqrt{\frac{s_{1}{ }^{2}}{n_{1}}+\frac{s_{2}^{2}}{n_{2}}}}
$$

where $x_{1}$ and $x_{2}$ are the two mean values being compared, $s_{1}$ and $s_{2}$ are the associated standard deviations and, $n_{1}$ and $n_{2}$ are the associated sample sizes for the two means.

Another variable needed for the t-test is the degrees of freedom (d.o.f.). This is calculated using the following equation, 


$$
\text { d. o. } f=\frac{\left(\frac{s_{1}{ }^{2}}{n_{1}}+\frac{s_{2}{ }^{2}}{n_{2}}\right)^{2}}{\left(\frac{s_{1}{ }^{4}}{n_{1}{ }^{2}\left(n_{1}-1\right)}+\frac{s_{2}{ }^{4}}{n_{2}{ }^{2}\left(n_{2}-1\right)}\right)}
$$

Using these two calculated values, the calculated t-statistic is compared to the critical t-value at the associated d.o.f. (Tables are available in all statistics textbooks). If the t-statistic is smaller than the critical t-value, the two means are statistically similar.

\section{Synthesis and characterization of gold nanoparticles.}

All glassware used for gold nanoparticle synthesis was cleaned using an aqua regia solution $\left(3: 1 \mathrm{v} / \mathrm{v}, \mathrm{HCl}: \mathrm{HNO}_{3}\right)$ and then thoroughly rinsed using deionized water.

\section{$6 \mathrm{~nm}$ gold nanoparticles}

The protocol developed by Slot et al. was used for the synthesis of the $6 \mathrm{~nm}$ gold nanoparticles. Gold (III) chloride $(33 \mu \mathrm{mol})$ was dissolved in $80 \mathrm{~mL}$ water and trisodium citrate $(34 \mu \mathrm{mol})$ was added to a separate $16 \mathrm{~mL}$ of deionized water along with $2.9 \mu \mathrm{mol}$ of tannic acid. Both solutions were heated to $60{ }^{\circ} \mathrm{C}$ and mixed together. Once a red colour was formed, the solution was heated to $95{ }^{\circ} \mathrm{C}$ and then cooled on ice.

The nanoparticles were then incubated with $\operatorname{bis}(p$ sulfonatophenyphosphine)phenyl phosphine (BSPP, $33 \mu \mathrm{mol}$ ), and allowed to stir for 12 hours. To the sample, solid sodium chloride was added in small portions until the solution changed colour due to the aggregation of the nanoparticles. The nanoparticles were then pelleted out using centrifugation (1000 RCF for 30 minutes), and resuspended in water. The nanoparticles were stored in the dark at $4{ }^{\circ} \mathrm{C}$. The synthesized nanoparticles were characterized using a transmission electron microscope and UV-Vis spectroscopy (Figure S1). 
(a)

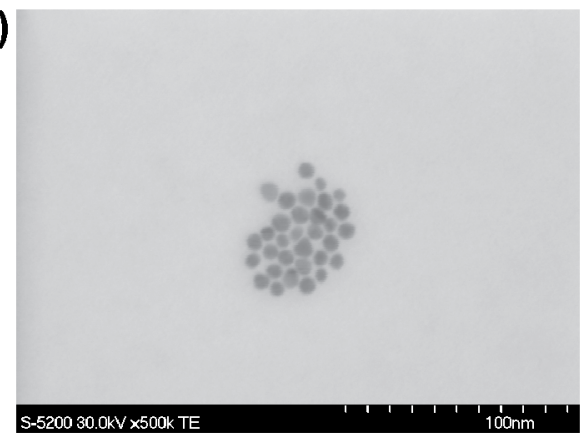

(b)

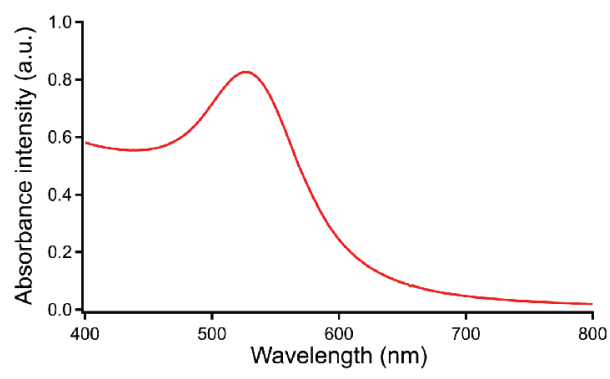

Figure S1. Characterization of $5.9 \pm 0.9 \mathrm{~nm}$ gold nanoparticles. (a) Transmission electron micrograph of $5.9 \mathrm{~nm}$ gold nanoparticles. (b) UV-Visible spectrum of $5.9 \mathrm{~nm}$ gold nanoparticles in water.

\section{Determination of gold nanoparticle concentration using UV-Vis analysis}

Nanoparticle concentration was determined using the procedure outlined by Haiss et al. ${ }^{3}$ Equation S1 was used to calculate the nanoparticle concentration using the size of the nanoparticle (Figure S1a) and the absorbance value at $450 \mathrm{~nm}$ (Figure S1b).

$$
N=\frac{A_{450} \times 10^{14}}{d^{2}\left[-0.295+1.36 e^{-\left(\frac{d-96.8}{78.2}\right)^{2}}\right]}
$$

where $\mathrm{N}$ corresponds to the number concentration of the nanoparticles (number of particles per $\mathrm{mL}$ ), $\mathrm{A}_{450}$ is the absorbance of the nanoparticles at $450 \mathrm{~nm}$, and $\mathrm{d}$ is the diameter of the nanoparticles as measured using the TEM images.

\section{Quantification of mPEG thiol}

Thiol functionalized mPEG (16.4 mg, $\left.\mathrm{M}_{\mathrm{n}} 6000 \mathrm{~g} \mathrm{~mol}^{-1}\right)$ was dissolved in $5 \mathrm{~mL}$ deionized water and varying amounts of solution was incubated with a constant aliquot of Aldrithiol (Reaction scheme provided in Figure S2). After two hours of reaction, the solutions were measured on a UV-Vis spectrometer, with the absorbance at $308 \mathrm{~nm}$ used for quantification. The 2-mercaptopyridone is known to have an extinction coefficient of $8080 \mathrm{M}^{-1} \mathrm{~cm}^{-1}$ at this wavelength. 

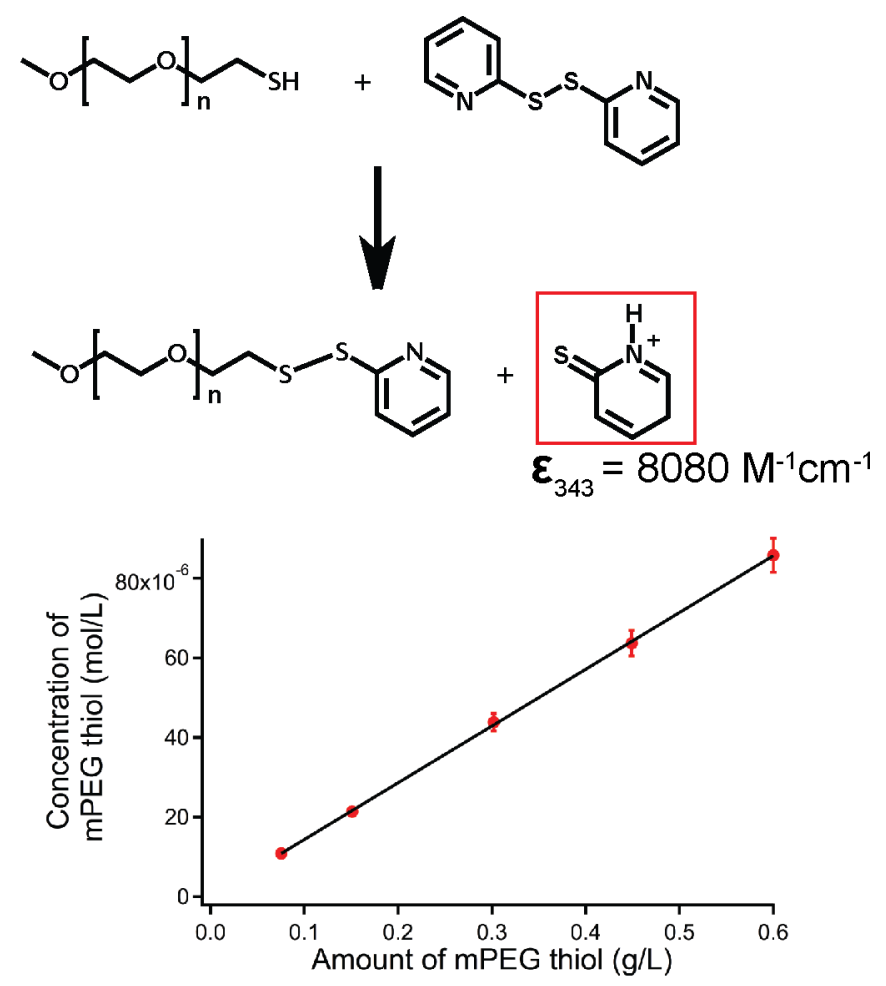

Figure S2. Determination of mPEG thiol concentration using 2-mercaptopyridone as a chromophoric label. (a) Reaction scheme demonstrating the reaction between the mPEG thiol and Aldrithiol. (b) Calibration curve used for the determination of mPEG thiol concentration. The $x$-axis contains the mass of $\mathrm{mPEG}$ thiol that was weighed out into a given volume of solution, and the y-axis consists of the concentration of thiol present in solution as measured using the data from UV-Vis analysis of 2-mercaptopyridine, which has an extinction coefficient of $8080 \mathrm{M}^{-1} \mathrm{~cm}^{-1}$ at $343 \mathrm{~nm}$.

\section{Influence of reaction temperature on the extent of reaction between gold nanoparticles and mPEG thiol.}

BSPP coated gold nanoparticles (diameter: $5.9 \mathrm{~nm}$ ) were incubated with varying amounts of mPEG thiol at two different temperatures: $25{ }^{\circ} \mathrm{C}$ and $50{ }^{\circ} \mathrm{C}$. After two hours of incubation, gel loading buffer was added to the different samples and then the samples were run on an agarose gel. (Figure S3 a and b) 
(a)

(i) (ii) (iii) (iv) (v) (vi)

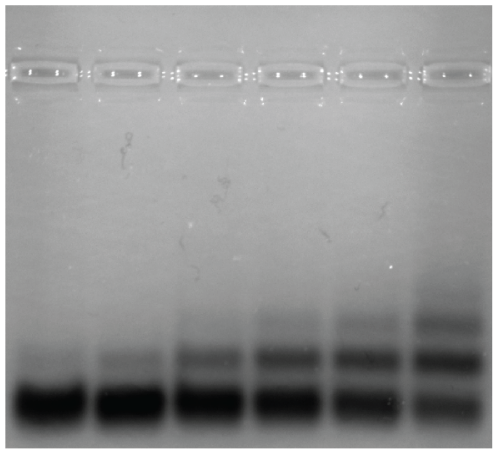

(b)

(i) (ii) (iii) (iv) (v) (vi)

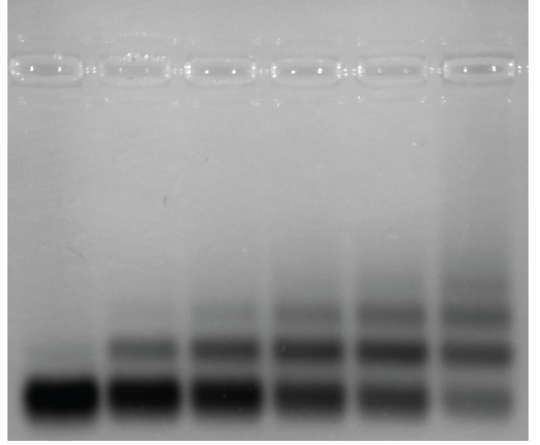

(c)

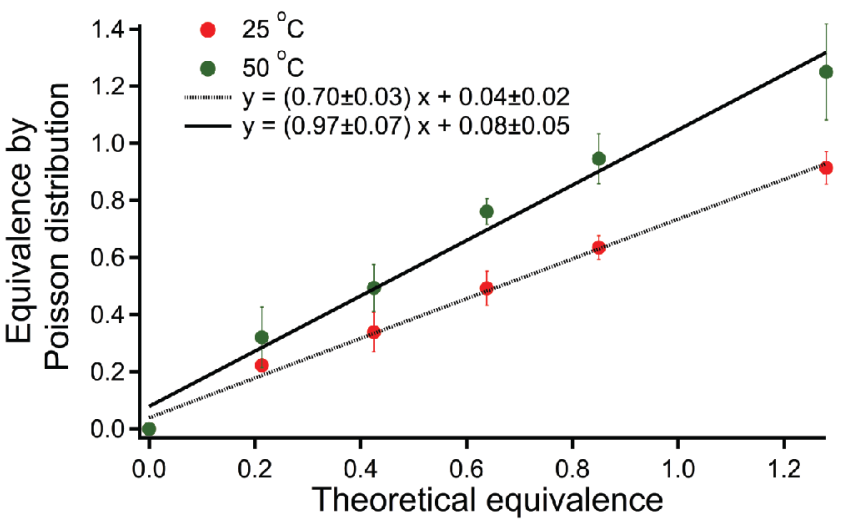

Figure S3. Influence of temperature on the extent of reaction between the gold nanoparticles and the mPEG thiol ligand. Agarose gel electrophoresis images were obtained for gold nanoparticle - mPEG thiol conjugates that were incubated at (a) $25^{\circ} \mathrm{C}$, and (b) $50^{\circ} \mathrm{C}$. The amounts of ligand (mol) used are: (i) 0 (ii) $3.75 \times 10^{-12}$ (iii) $7.50 \times 10^{-12}$ (iv) $1.13 \times 10^{-11}(\mathrm{v}) 1.50 \times 10^{-11}$ (vi) $2.25 \times 10^{-11}$. (c) Correlation curves used to determine the extent of correlation between the amount of ligand incubated per nanoparticle and the equivlance measured from the statistical distribution of nanoparticle - mPEG thiol conjugates.

\section{Evaluation of the precision in the distribution of conjugates}

Six different samples of the BSPP coated gold nanoparticles, from a single batch, were incubated with the same quantity of ligand for at least 2 hours. These samples were then analyzed using agarose gel electrophoresis, with the resulting image shown in Figure S4. 
(a)

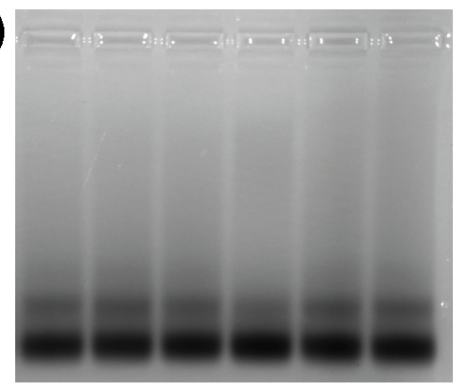

(c)

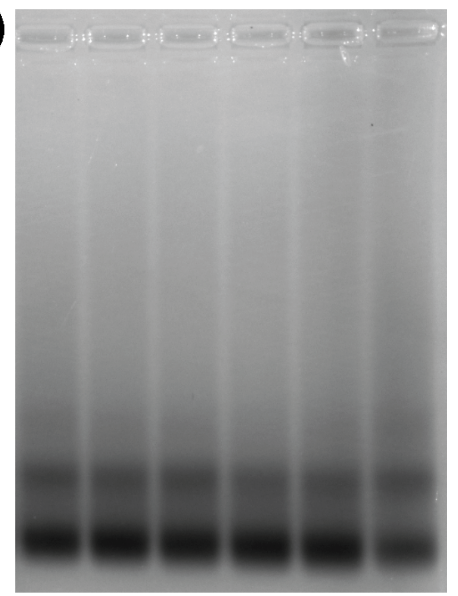

(b)

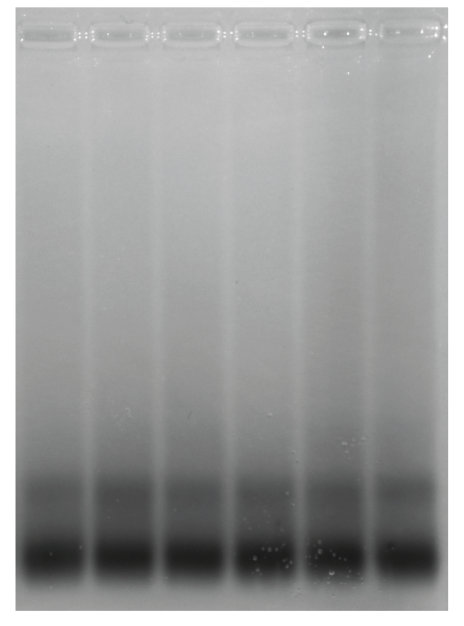

Figure S4. Evaluation of assay precision. Agarose gel electrophoretic analysis of replicate samples of gold nanoparticles incubated with specific amounts of mPEG thiol ligand (mol) (a) $1.6 \times 10^{-11}$, (b) $2.3 \times 10^{-11}$ and (c) $3.5 \times 10^{-11}$

\section{AuNP-DNA conjugate distributions to determine nanoparticle concentration}

BSPP coated gold nanoparticles were incubated with varying equivalents of thiol functionalized DNA (56-mer; IDT DNA, Coraville, IA) for two hours at room temperature. A solution with an ionic strength of $0.125 \mathrm{M} \mathrm{NaCl}$ was used to ensure minimal electrostatic repulsion between the gold nanoparticle and the DNA strand. The DNA functionalized gold nanoparticles were subsequently incubated with $50 \mathrm{nmol}$ of mPEG800 thiol (Sigma-Aldrich, Oakville, ON, Canada) for 30 minutes at room temperature. The samples were then analyzed on a $3 \%$ agarose gel in $0.5 \times$ TBE at an electric field strength of $5.7 \mathrm{~V} \mathrm{~cm}^{-1}$. An image of the agarose gel is shown in Figure S5a. 

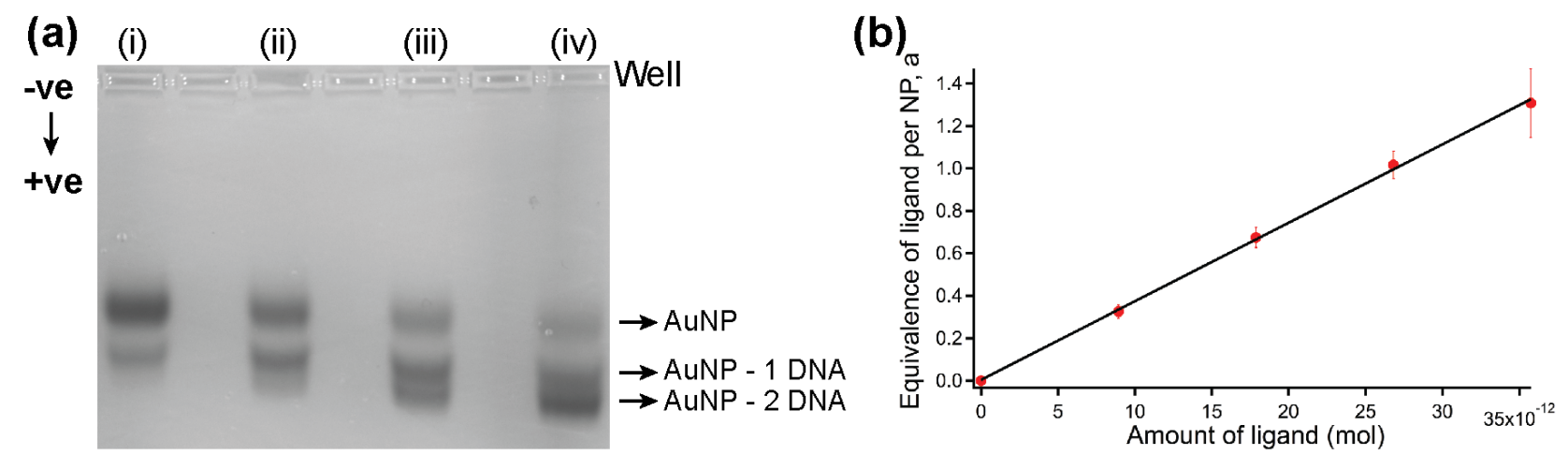

Figure S5 Gel electrophoretic analysis of gold nanoparticle - nucleic acid conjugates for the purpose of quantifying gold nanoparticle concentration in solution. (a) Gold nanoparticles incubated with varying amounts of DNA (mol) (i) $9.93 \times 10^{-12}$ (ii) $1.99 \mathrm{x}$ $10^{-11}$ (iii) $2.98 \times 10^{-11}$ (iv) $3.97 \times 10^{-11}$. A $3 \%$ agarose gel was used in $0.5 \times \mathrm{TBE}$ and run at $5.7 \mathrm{~V} \mathrm{~cm}^{-1}$. (b) A calibration curve representing the relationship between the amount of ligand added to a fixed quantity of gold nanoparticle solution and the equivalence of ligand per nanoparticle as predicted by the Poisson model, which was applied to the distribution of conjugates seen in the gel in part (a). The error bars on the points correspond to 1 standard deviation.

A linear curve was fit to the calibration points in Figure S5b, and the amount of ligand corresponding to a ligand equivalence of 1 was calculated from the equation of the line. Using this information, the concentration of the gold nanoparticles was calculated to be $2.7 \pm 0.1 \mu \mathrm{M}$. This represents a $4 \%$ deviation from the concentration predicted using $\mathrm{UV}-\mathrm{Vis}$ analysis of the sample of gold nanoparticles $(2.8 \pm 0.2 \mu \mathrm{M})$.

\section{UV-Vis analysis of binary QD (CdSe/ZnS; core/shell)}

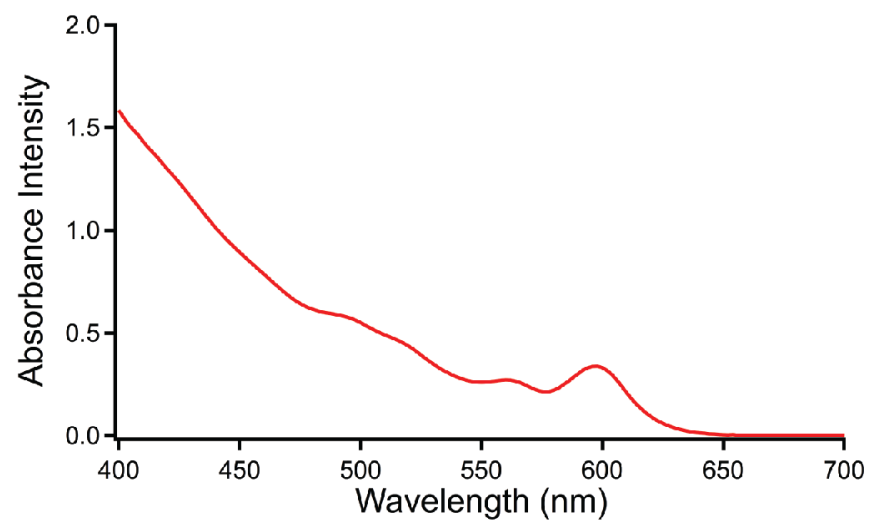

Figure S5. Absorbance spectra of quantum dots with a core composition of CdSe and a $\mathrm{ZnS}$ shell. 


\section{Hexahistidine functionalized mPEG}

The thiol functionalized poly(ethylene glycol) methyl ether (20 mg, $3.33 \mu \mathrm{mol})$ was incubated with TCEP $(33 \mu \mathrm{mol})$ for 2 hours to reduce any disulfide to the thiol form. Excess TCEP was removed using a NAP-5 desalting column, and the thiol mPEG was then incubated with the maleimide-functionalized peptide $(33 \mu \mathrm{mol})$ for 24 hours. Unreacted peptide was removed using a NAP-5 desalting column. The sample was then purified using a His GraviTrap column to remove any mPEG that may not be functionalized with the peptide. The sample was then desalted again to remove excess imidazole, and then quantified as described below.

\section{Quantification of hexahistidine functionalized $m P E G$}

The protocol outlined by Gong et al. was used to quantify the amount of hexahistidine functionalized mPEG in solution. To prepare a calibration curve, the disulfide protected PEGs described above was utilized. These PEG molecules were incubated with barium chloride and iodine. A $0.24 \mathrm{M}$ barium chloride solution was prepared in $1 \mathrm{M}$ hydrochloric acid. A 0.1 M iodine-potassium iodide solution was prepared in deionized water. To an aliquot of PEG solution $(400 \mu \mathrm{L})$ at different concentrations, $100 \mu \mathrm{L}$ of the barium chloride solution was added along with $50 \mu \mathrm{L}$ of the iodine solution. After a 30 -minute incubation, the solution was centrifuged to pellet the precipitate, and the supernatant was measured on a UV-Vis spectrophotometer. A calibration curve was constructed based on known amounts of mPEG, and the concentration of the hexahistidine functionalized mPEG was determined using linear regression analysis. 


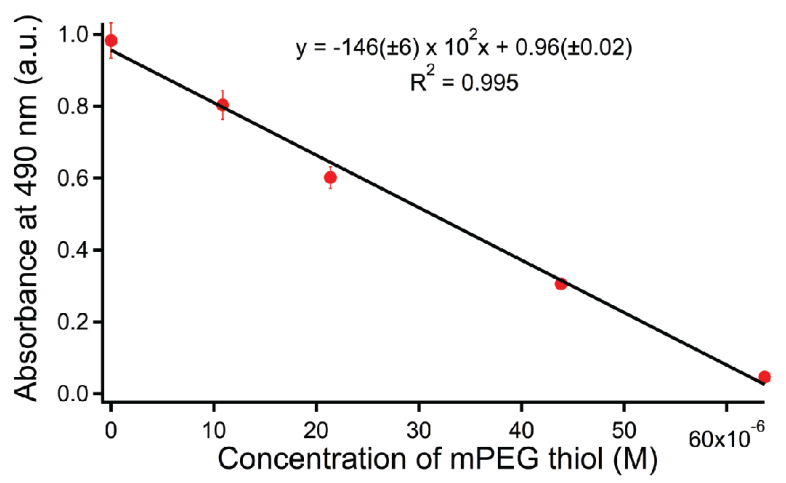

Figure S6. Quantification of mPEG HisTag using a complexation reaction between the ether groups of the polymer and barium iodide. For the calibration curve, the amount of mPEG in solution was established as described above for quantifying mPEG thiol.

\section{UV-Vis analysis of alloyed semiconductor nanocrystals.}

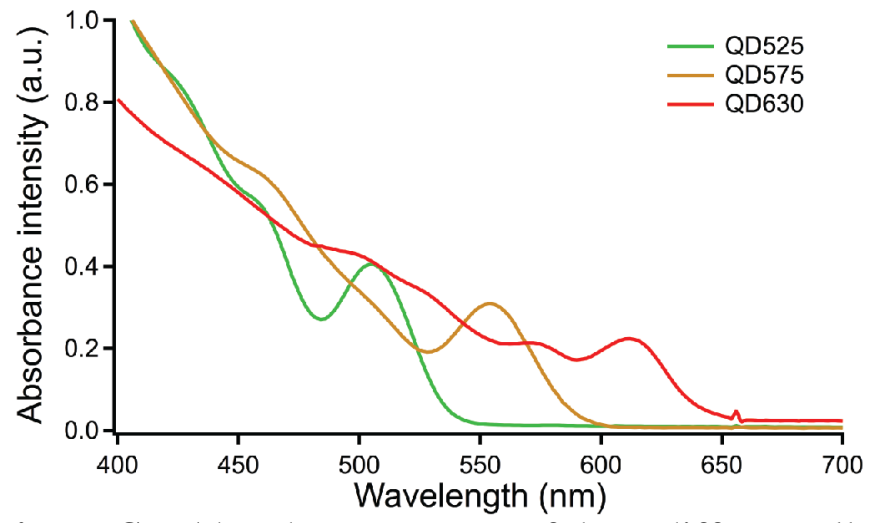

Figure S7. Absorbance spectra of three different alloyed quantum dots with different emission wavelengths.

\section{Synthesis of monovalent QD-DNA conjugates}

The procedure for the synthesis of the monovalent QD-DNA conjugates was previously reported. Briefly, mPEG750 coated QD525 were incubated with a low equivalence of DNA $(<1)$, that was functionalized with a HisTag. After incubating for 4 hours, the QD-DNA conjugates were captured onto diethylaminoethanol functionalized magnetic beads. The QD-DNA monoconjugates were then selectively eluted using wash 
solutions of a specific ionic strength. The isolation of QD-DNA monoconjugates was verified using agarose gel electrophoresis (Figure S6a).

The nucleic acid conjugated onto the QD525 was also labeled with Cy5. The monovalency of the QD-DNA conjugate allows the quantification of the QD525 using the absorbance spectra of $\mathrm{Cy} 5$ as they are present in a 1:1 ratio (Figure S6b).

(a)

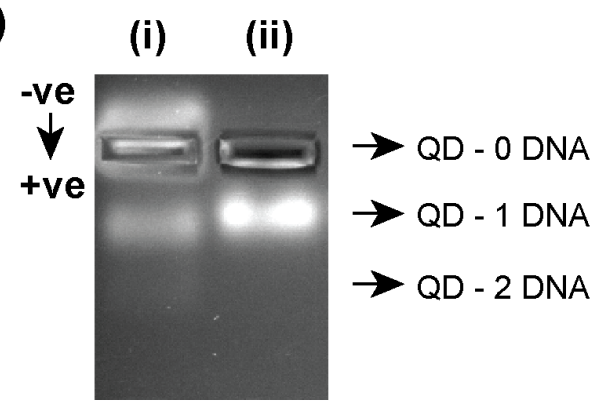

(b)

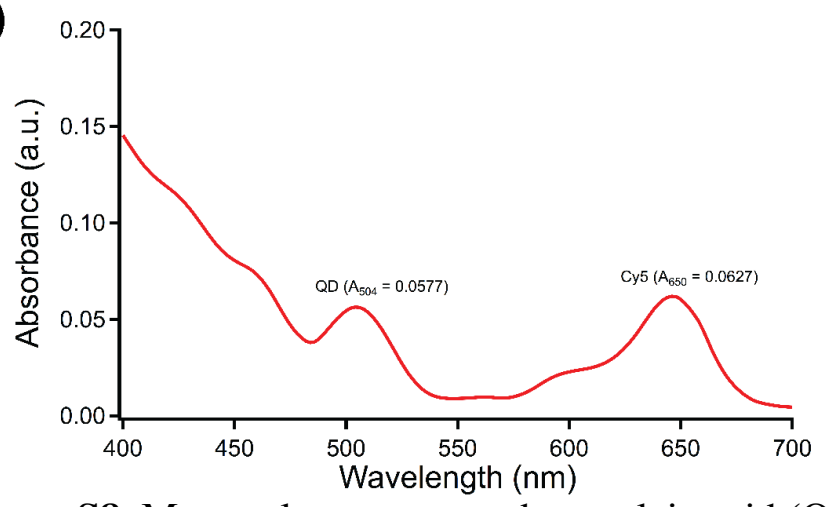

Figure S8. Monovalent quantum dot-nucleic acid (QD-DNA) conjugates (a) Agarose gel electrophoresis used to confirm successful isolation of QD-DNA conjugates. Lane (i) represents QD-DNA conjugates of multiple valences, Lane (ii) represents the QD-DNA monoconjugates that were isolated using the magnetic bead protocol. (b) UV-Vis analysis of QD-DNA monoconjugates, where the DNA was also labeled with Cy5.

\section{References}

(1) WaveMetrics.

(http://www.wavemetrics.com/products/igorpro/dataanalysis/curvefitting.htm). Accessed on 04 December 2014.

(2) T-Test; Stone, D.; Ellis, J., Eds. (http://www.chem.utoronto.ca/coursenotes/analsci/StatsTutorial/ttest.html). Accessed on 04 December 2014.

(3) Haiss, W.; Thanh, N. T. K.; Aveyard, J.; Fernig, D. G. Anal. Chem. 2007, 79, 4215-4221. 